\title{
Prediction of Combustion Noise in a Model Combustor Using a Network Model and a LNSE Approach
}

\author{
Wolfram C. Ullrich* \\ Lehrstuhl für Thermodynamik \\ Department of Mechanical Engineering \\ Technische Universität München \\ 85748 Garching \\ Germany \\ ullrich@td.mw.tum.de \\ Yasser Mahmoudi \\ Department of Engineering \\ University of Cambridge \\ Cambridge CB2 1PZ \\ United Kingdom \\ sm2027@cam.ac.uk \\ Kilian Lackhove \\ Fachgebiet für Energie- \\ und Kraftwerkstechnik \\ Technische Universität Darmstadt \\ 64287 Darmstadt \\ Germany \\ lackhove@ekt.tu-darmstadt.de
}

André Fischer

Rolls-Royce Deutschland Ltd \& Co KG

ES-2 Turbine, Combustion Subsystems

15827 Blankenfelde-Mahlow

Germany

Andre.Fischer@Rolls-Royce.com

Christoph Hirsch, Thomas Sattelmayer

Lehrstuhl für Thermodynamik

Department of Mechanical Engineering

Technische Universität München

85748 Garching

Germany

hirsch@td.mw.tum.de, sattelmayer@td.mw.tum.de 


\author{
Ann P. Dowling, Nedunchezhian Swaminathan \\ Department of Engineering \\ University of Cambridge \\ Cambridge CB2 1PZ \\ United Kingdom \\ apd1@cam.ac.uk,ns341@cam.ac.uk
}

\author{
Max Staufer \\ Rolls-Royce Deutschland Ltd \& Co KG \\ ES-2 Turbine, Combustion Subsystems \\ 15827 Blankenfelde-Mahlow \\ Germany \\ Max.Staufer@Rolls-Royce.com
}

\begin{abstract}
The reduction of pollution and noise emissions of modern aero engines represents a key concept to meet the requirements of the future air traffic. This requires an improvement in the understanding of combustion noise and its sources, as well as the development of accurate predictive tools. This is the major goal of the current study where the LOTAN network solver and a hybrid CFD/CAA approach are applied on a generic premixed and pressurized combustor to evaluate their capabilities for combustion noise predictions. LOTAN solves the linearized Euler equations (LEE) whereas the hybrid approach consists of RANS mean flow and frequency-domain simulations based on linearized Navier-Stokes equations (LNSE). Both solvers are fed in turn by three different combustion noise source terms which are obtained from the application of a statistical noise model on the RANS simulations and a postprocessing of an incompressible and compressible LES. In this way the influence of the source model and acoustic solver is identified. The numerical results are compared with experimental data. In general good agreement with the experiment is found for both the LOTAN and LNSE solvers. The LES source models deliver better results than the statistical noise model with respect to the amplitude and shape of the heat release spectrum. Beyond this it is demonstrated that the phase relation of the source term does not affect the noise spectrum. Finally, a second simulation based on the inhomogeneous Helmholtz equation indicates the minor importance of the aerodynamic mean flow on the broadband noise spectrum.
\end{abstract}

\title{
Nomenclature
}

A Area, $\mathrm{m}^{2}$

$c$ Speed of sound, $\mathrm{m} / \mathrm{s}$

$c_{p}, c_{v} \quad$ Specific isobaric and isochoric heat capacities, $\mathrm{J} /(\mathrm{kgK})$

$\delta_{f} \quad$ Flame thickness in network model, $\mathrm{m}$

$f$ Frequency, $\mathrm{Hz}$

$\mathcal{F}$ Fourier transformation, $1 / \mathrm{Hz}$

$h_{f, k}^{0} \quad$ Enthalpy of formation of $k$-th species, $\mathrm{J} /(\mathrm{kgK})$

$\eta$ Dynamic viscosity, $\mathrm{kg} /(\mathrm{m} \mathrm{s})$

$H_{u} \quad$ Lower heating value, $\mathrm{J} / \mathrm{kg}$

i Imaginary unit

$k$ Wave-number, $1 / \mathrm{m}$

$\kappa$ Isentropic exponent

$l$ Length, $\mathrm{m}$

$\lambda$ Thermal conductivity, $\mathrm{W} /(\mathrm{m} \mathrm{K})$

M Mach number

$\dot{m}$ Mass flow rate, $\mathrm{kg} / \mathrm{s}$

n Surface normal

$p$ Pressure, $\mathrm{N} / \mathrm{m}^{2}$

$\phi$ Arbitrary field quantity

*Address all correspondence to this author. 


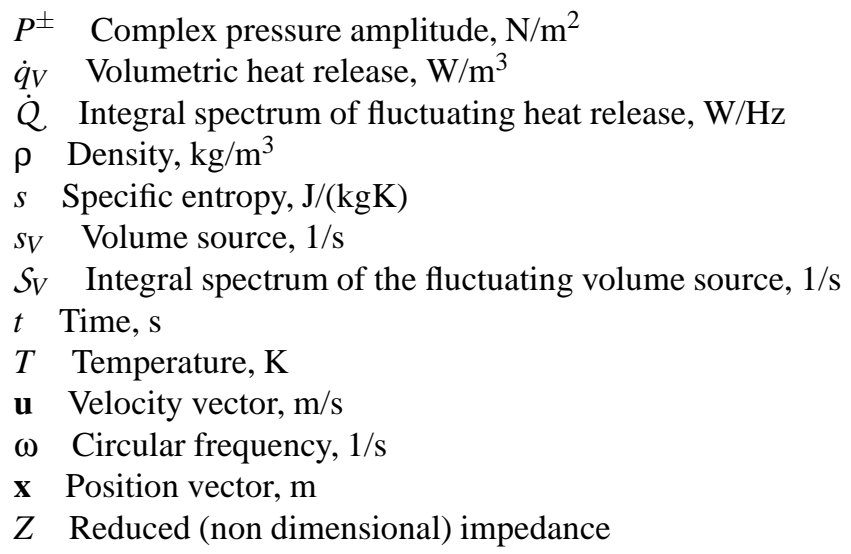

\section{Introduction}

In the near future the air traffic is expected to increase significantly which makes a further reduction of aircraft noise indispensable. This must address all aircraft components, but particularly the engine, in order to meet strict political requirements such as the $\mathrm{ACARE}^{1}$ goal prescribing a noise reduction of $50 \%$ by 2020 . Combustion noise is becoming increasingly important as a major noise source in aircraft. This is partially because advances in the design of aircraft have reduced other noise sources such as jet, fan and external aerodynamic noise [1]. Furthermore, modern low-NOx combustors show a considerable increase in noise emission [2]. This is because lean premixed and stratified combustion burns more unsteadily. The origin of combustion noise can be found in two different effects [3]: The first one, the direct noise, is caused by heat release fluctuations of the flame, which can be due to broadband stochastic turbulent fluctuations or mixture fluctuations at the flame. Secondly, the indirect noise originates from the acceleration of convected temperature (hot-spots) and vortical fluctuations in the turbine, which are also created by unsteady combustion [4].

In the past analytical methods, large eddy simulations (LES) and hybrid CFD/CAA-methods (computational fluid dynamics/ computational aeroacoustics) were developed in order to determine the combustion noise of turbulent confined flames. Analytical models introduce crucial simplifications with respect to the geometry and flow field which limit their validity to simple cases. For this reason complex geometries are represented by series of analytical elements which are linked through network models. The capabilities of compressible LES for accurate combustion noise predictions were demonstrated in laboratory scale geometries as well as full scale engines, which requires, however, excessive computational effort [5,6]. An intermediate model between network modeling approaches and unsteady Reynolds-averaged Navier-Stokes (RANS) simulations or even compressible LES are the so-called hybrid approaches. On the one hand they require far less computational effort than compressible LES, but maintain a higher level of physics than network models. Hybrid methods rely on the decomposition of the flow field into a stationary mean part around which small amplitude fluctuations propagate. This can be either described by the linear wave equation, linearized Euler (LEE) and Navier-Stokes equations (LNSE) or acoustic perturbation equations (APE) [7]. These equations incorporate combustion noise source terms which are either directly evaluated from LES [8] and unsteady RANS (URANS) simulations, or are subject to statistical modeling approaches [9, 10]. The main advantage of statistical modeling is the low computational effort of steady RANS simulations, while the major issue represents the combustion noise source reconstruction and their frequency content. The most prominent models are the Fast Random Particle-Mesh Methods (FRPM) [9-11] and the model developed by Hirsch et al. [12-14].

The goal of the present study is to assess the capabilities of the Low-Order Thermo-Acoustic Network model (LOTAN) and the hybrid RANS/LNSE-approach for the prediction of combustion noise in the generic CESAM-HP combustor, installed at the EM2C laboratory at the Centre National de la Recherche Scientifique (CNRS). This study is focused on the direct combustion noise, whereas the role of entropy waves and the indirect noise generation is only briefly discussed and a topic of further studies. The CESAM-HP test rig basically consists of a confined pressurized, swirled and premixed propane flame, which is operated under lean conditions. Both propagation models are solved in frequency domain and are excited by combustion noise source spectra obtained from three different approaches with different levels of complexity. More specifically, the simplest method is the statistical noise model, which was initially developed by Hirsch et al. [12] and recently extended by Liu et al. [2]. It utilizes the turbulence quantities of the RANS to compute the heat release spectrum. In the other two models the source terms are determined by postprocessing the results of the incompressible LES solver PRECISE-UNS [15, 16] and the compressible LES solver AVBP [17]. The AVBP data were provided by CERFACS [18, 19].

\footnotetext{
${ }^{1}$ Advisory Council for Aviation Research and Innovation in Europe, www.acare4europe.com
} 
The resulting pressure spectra in the combustor are compared against each other, and also compared with experimental measurements conducted and provided by CNRS [20,21]. In this way the influence of the acoustic propagation model and the source term on the resulting pressure spectrum can be identified and separated, which are the two major goals of this study. Both the LOTAN and LNSE solver have already demonstrated their capabilities for accurate combustion noise predictions in academic cases [1,2] as well as industry-relevant combustor geometries [22,23]. Moreover, LOTAN and LNSE were already validated against each other in the frame of a simple model combustor [24]. In this study the complex CESAM-HP combustor is considered. The acoustic model of the LNSE solver of the CESAM-HP combustor is based on previous studies where the LNSE were applied in conjunction with a statistical noise model $[12,25]$.

The paper is structured as follows: First the basic equations concerning the LNSE and LOTAN solver are briefly reviewed. Then the CESAM-HP combustor test case as well as the corresponding numerical simulation models are introduced. This is followed by the presentation and discussion of the results with particular focus on the comparison between the solvers and source models. Finally the study is summarized and conclusions concerning the present and future work are given.

\section{Governing Equations}

\subsection{Linearized Navier-Stokes Equations (LNSE)}

The different length and time scales of the fluid-dynamical and acoustic quantities allow to split up the unsteady flow field into its statistically stationary mean part and small amplitude fluctuations in time and space, i.e. $\phi(\mathbf{x}, t)=\bar{\phi}(\mathbf{x})+\phi^{\prime}(\mathbf{x}, t)$. The LNSE are derived by inserting this approach into the continuity, momentum and energy conservation equations and neglecting products of the fluctuating quantities. The transformation of these equations into frequency domain is achieved by assuming time-harmonic fluctuations with $\phi^{\prime}(\mathbf{x}, t)=\operatorname{Re}\{\hat{\phi}(\mathbf{x}) \exp (\mathrm{i} \omega t)\}$ and $\partial \phi^{\prime} / \partial t=\operatorname{Re}\{\operatorname{i} \omega \hat{\phi}(\mathbf{x}) \exp (\mathrm{i} \omega t)\}, \operatorname{yielding}$

$$
\begin{aligned}
& \mathrm{i} \omega \hat{\rho}+\overline{\mathbf{u}} \nabla \hat{\rho}=-\hat{\mathbf{u}} \nabla \bar{\rho}-\bar{\rho} \nabla \cdot \hat{\mathbf{u}}-\hat{\rho} \nabla \cdot \overline{\mathbf{u}}+\bar{\rho} \hat{s}_{V} \\
& \mathrm{i} \omega \bar{\rho} \hat{\mathbf{u}}+\bar{\rho} \overline{\mathbf{u}} \nabla \hat{\mathbf{u}}=-\bar{\rho} \hat{\mathbf{u}} \nabla \overline{\mathbf{u}}-\hat{\rho} \overline{\mathbf{u}} \nabla \overline{\mathbf{u}}-\nabla \hat{p}+\eta\left[\Delta \hat{\mathbf{u}}+\frac{1}{3} \nabla(\nabla \cdot \hat{\mathbf{u}})\right] \\
& \mathrm{i} \omega \hat{p}+\overline{\mathbf{u}} \nabla \hat{p}=-\hat{\mathbf{u}} \nabla \bar{p}-\kappa \bar{p} \nabla \cdot \hat{\mathbf{u}}-\kappa \hat{p} \nabla \cdot \overline{\mathbf{u}}+\bar{\rho} \bar{c}^{2} \hat{s}_{V}+(\kappa-1) \hat{\dot{q}}_{V}+(\kappa-1)\left\{2 \eta\left[\nabla \overline{\mathbf{u}}: \nabla \hat{\mathbf{u}}+\nabla \overline{\mathbf{u}}: \nabla \hat{\mathbf{u}}^{T}-\frac{2}{3}(\nabla \cdot \overline{\mathbf{u}})(\nabla \cdot \hat{\mathbf{u}})\right]+\lambda \Delta \hat{T}\right\} .
\end{aligned}
$$

In this study the LNSE Eqs. (1) - (3) are solved successively for the fluctuating density $\hat{\rho}$, velocities $\hat{\mathbf{u}}$ and pressure $\hat{p}$ with the frequency $\omega=2 \pi f$ as an arbitrary parameter. The mean flow quantities $\bar{\rho}, \overline{\mathbf{u}}$ and $\bar{p}$ are assumed to be given and constant. The ratio of the isobaric and isochoric heat capacities $c_{p}$ and $c_{v}$ is defined as the isentropic exponent $\kappa$, while viscous and thermal diffusion are quantified by $\eta$ and $\lambda$, respectively. The LNSE are excited parametrically either by the fluctuating heat of combustion $\hat{\dot{q}}_{V}$ or by the volume source terms $\hat{s}_{V}$. The heat of combustion is characterized by fluctuations of the species composition and given by $\hat{\dot{q}}_{V}=-\sum_{k=1}^{N} h_{f, k}^{0} \hat{\dot{\omega}}_{k}$, with $h_{f, k}^{0}$ and $\hat{\dot{\omega}}_{k}$ being the enthalpy of formation and the formation rate of the $k$-species, respectively. The volume sources describe fluctuations of the reaction progress variable and are not necessarily accompanied by the generation of entropy fluctuations. The volume source term and fluctuating heat of combustion are related to each other by assuming an incompressible low-Mach number flame, leading to the following relation [26]

$$
\hat{s}_{V}=\frac{\kappa-1}{\bar{\rho} \bar{c}^{2}} \hat{\dot{q}}_{V}
$$

In general the LNSE feature both the propagation of acoustic waves as well as the convective vorticity $\hat{\boldsymbol{\Omega}}$ and entropy waves $\hat{s}[27]$ which may be computed in the postprocessing by evaluating

$$
\hat{\mathbf{\Omega}}=\nabla \times \hat{\mathbf{u}}, \quad \hat{s}=c_{v} \frac{\hat{p}}{\bar{p}}-c_{p} \frac{\hat{\rho}}{\bar{\rho}}
$$

Unlike the hybrid approach, LOTAN is based on the LEE which are easily obtained setting the viscous and thermal diffusion to zero $(\eta=\lambda=0)$ in the Eqs. (1) - (3).

\subsection{Inhomogeneous Helmholtz equation}

In order to separate the influence of the aerodynamic mean flow field on the combustion noise spectrum the simulations are additionally performed with the inhomogeneous Helmholtz equation. It is derived by neglecting the aerodynamic mean 
flow $(\overline{\mathbf{u}}=0, \nabla \overline{\mathbf{u}}=\nabla \bar{\rho}=\nabla \bar{p}=0)$, diffusive effects $(\eta=\lambda=0)$ and volume sources $\left(\hat{s}_{V}=0\right)$ in the LNSE Eqs. (1) - (3). The combination of the divergence of the linearized momentum Eq. (2) and the time derivative of the energy Eq. (3) finally yields [28]

$$
\nabla \cdot\left(\frac{1}{\bar{\rho}} \nabla \hat{p}\right)+\frac{1}{\bar{\rho}} k^{2} \hat{p}=-\mathrm{i} \omega \frac{\kappa-1}{\bar{\rho} \bar{c}^{2}} \hat{\dot{q}}_{V}
$$

where $k=\omega / \bar{c}$ is the acoustic wave number and $\bar{c}$ the speed of sound of an ideal gas. This equation solely takes into account the mean thermodynamic properties $\bar{\rho}$ and $\bar{c}$.

\subsection{Boundary Conditions}

The acoustic waves entering the domain are described using the reduced acoustic impedance $Z:=\hat{p} /(\hat{\mathbf{u}} \cdot \mathbf{n} \bar{\rho} \bar{c})$ which directly leads to a boundary condition of the following form:

$$
(\hat{\mathbf{u}} \cdot \mathbf{n}) Z \bar{\rho} \bar{c}-\hat{p}=0 .
$$

Stationary, acoustic hard walls are given by $Z \rightarrow \infty$, while pressure outlets are defined setting $Z=0$. In the case of the inhomogeneous Helmholtz Eq. (6) the impedance BC Eq. (7) is exclusively expressed in terms of the acoustic pressure by exploiting the simplified momentum Eq. (2) $i \omega \bar{\rho} \hat{\mathbf{u}}=-\nabla \hat{p}$ which yields

$$
(\nabla \hat{p} \cdot \mathbf{n}) Z \bar{c}-\mathrm{i} \omega \hat{p}=0
$$

\subsection{Combustion Noise Source Terms}

In this study LOTAN and the LNSE are each excited using three different combustion noise sources which are given in terms of an integral heat release spectrum $\dot{Q}$ and which are obtained as follows:

\subsubsection{Statistical noise model by Hirsch/Liu.}

This model is applied in the postprocessing of stationary compressible RANS simulations which are described in the next section. The core idea of this model is to represent the flame as an assembly of individual local sound sources, in which an assumed turbulence spectrum is auto-correlated and transformed into frequency domain by postulating equal energy contents in wave number and frequency space. The total heat release is obtained by the integration of each individual sound source contribution over the total flame volume while taking the spatio-temporal coherence into account using the concept of the coherence volume $[2,13]$.

\subsubsection{Incompressible LES (PRECISE-UNS).}

The source spectrum was also computed by means of an incompressible LES based on the Favre-averaged NavierStokes equations which are closed by a dynamic Smagorinsky subgrid turbulence model. Combustion is accounted for by the Flamelet Generated Manifold (FGM) method along with premixed flamelets using the GRI-3.0 mechanism. Furthermore the Artificially Thickened Flame (ATF) procedure is applied. More details about the simulation model can be found in [29]. The heat release source spectrum is retrieved in the postprocessing by the evaluation of the fast Fourier transformed $\mathcal{F}\{\ldots\}$ time derivative of the density [8]

$$
\bar{\rho} \mathcal{S}_{V}(f)=\mathcal{F}\left\{\int_{V} \frac{\partial \rho}{\partial t} \mathrm{~d} V\right\}
$$

where $\mathcal{S}_{V}(f)$ is the integral spectrum of the volume source term. To decrease the computational effort, the volume integral is reduced to a surface integral by exploiting the divergence theorem in Eq. (9) giving

$$
\bar{\rho} \mathcal{S}_{V}(f)=-\mathcal{F}\left\{\int_{A_{2}}(\rho \mathbf{u} \cdot \mathbf{n})_{2} \mathrm{~d} A+\int_{A_{1}}(\rho \mathbf{u} \cdot \mathbf{n})_{1} \mathrm{~d} A\right\}
$$

The subscripts 1 and 2 indicate the combustor inlets and nozzle cut plane at $x=0.173 \mathrm{~m}$, respectively. The volume source spectrum is transformed to an equivalent heat release spectrum by exploiting Eq. (4) [29]. 


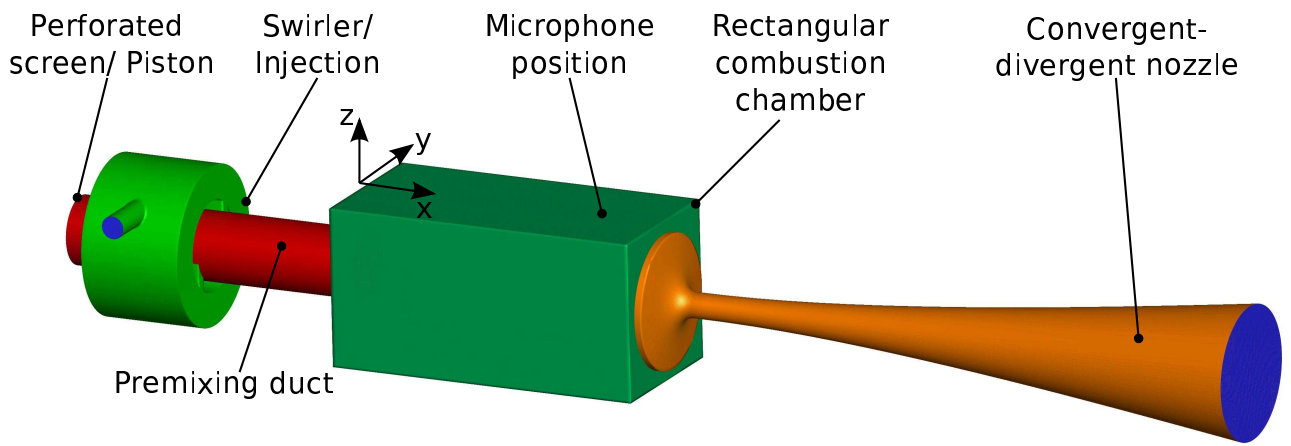

Fig. 1. CNRS CESAM-HP combustor model

\subsubsection{Compressible LES (AVBP).}

The compressible LES was jointly performed by CERFACS and CNRS $[18,19,21]$ using the solver AVBP developed by CERFACS [17]. Similar to the incompressible LES postprocessing the integral spectrum is obtained by the mass flux evaluation in Eq. (10). It should be emphasized that the evaluation of the compressible LES delivers a spectrum which is not only a pure source term, but also contains feedback and coupling effects between the flame and acoustics, which lead under certain conditions to thermoacoustic instabilities [30]. These coupling effects can be described in terms of a flame transfer function (FTF), which is, however, not available from experimental measurements or LES computations. Consequently the different contributions in the spectrum cannot be easily separated. When using the LOTAN and LNSE model, it is assumed that this term is a pure source term which acts independent from the acoustics. However we keep in mind that the resulting pressure spectra may also contain the feedback effect between the flame and acoustic waves.

\section{Numerical Setup}

\subsection{Configuration}

The CESAM-HP test rig represents a rectangular combustion chamber with length of $l_{c}=0.14 \mathrm{~m}$, which is connected with the atmosphere via a convergent-divergent nozzle mounted at the downstream end of the combustor [31]. A straight duct with length of $l_{d}=0.1345 \mathrm{~m}$ and a swirler are installed at the upstream side in order to inject and to twist the mixture of propane and air. Depending on the operating point additional pure air is injected through a perforated screen, which is part of a tunable damper. An air jet can be introduced in the center of the perforated screen to modify the vortex dynamics of the swirler. The setup is sketched in Fig. 1. The point of origin $x=0 \mathrm{~m}$ in axial direction $x$ is defined at the area jump between duct and combustor, called combustor face plate.

In the experiment three different operating points are defined by variations of the air mass flow rates through the swirler, perforated screen and axial jet inlet. The chamber is operated under lean conditions with fixed global equivalence ratio of 0.85 each time. In the present study only one out of three operating points is investigated, which is referred here to as OP13-5-0 and specified in Tab. 1. This operating points features the most stable combustion conditions so that thermoacoustic instabilities are less present.

\subsection{Low-Order Thermo-Acoustic Network Model (LOTAN)}

LOTAN has already been used extensively and is described in previous publications, e.g. [30,32,33]. LOTAN solves the LEE for axial and circumferential fluctuations (Eqs. (1) - (3) with $\eta=\lambda=0$ ) propagating in cylindrical or thin-annular geometries while radial fluctuations are neglected, i.e. $\phi^{\prime}(x, \theta, t)=\operatorname{Re}\{\hat{\phi}(x) \exp (\mathrm{i} \omega t+\mathrm{i} n \theta)\}[32,34]$. The mean flow is assumed to be one-dimensional and homogeneous in straight duct sections implying $\nabla \overline{\mathbf{u}}=\nabla \bar{\rho}=\nabla \bar{p}=0$ in Eqs. (1) - (3). The model is formulated in terms of a network of modules describing the features of the geometry, such as straight ducts, area changes and heat source modules. The application of inlet and outlet boundary conditions then enables determination of the linear perturbations. The network model of the CNRS combustor is sketched in Fig. 2.

For duct modules of constant cross-sectional area and no heat input $\left(\hat{\dot{q}}_{V}=0\right)$, acoustic wave propagation and convection of entropy and vorticity waves are used to relate the perturbations at one end of the duct to those at the other. In case of the CNRS test rig only one-dimensional acoustic and entropy wave propagation is considered $(n=0)$, which is described by the 
Table 1. Examined operating point OP-13-5-0

\begin{tabular}{cl}
\hline Quantity & Value \\
\hline Swirler air mass flow (g/s) & 13 \\
Axial ICS/piston air mass flow (g/s) & 0 \\
Axial jet air mass flow (g/s) & 5 \\
Mean static pressure (bar) & 2.43 \\
Total temperature inlet (K) & 296.5 \\
Total mass flow (g/s) & 18.983 \\
Swirler fuel mass flow (g/s) & 0.983 \\
Equivalence ratio (-) & 0.85 \\
Thermal power $(\mathrm{W})$ & 45,522 \\
\hline
\end{tabular}

solution of the one-dimensional LEE [35]:

$$
\begin{aligned}
& \hat{p}(x)=P^{+} e^{-\mathrm{i} k \frac{x}{1+M}}+P^{-} e^{+\mathrm{i} k \frac{x}{1-M}} \\
& \hat{u}(x)=\frac{1}{\bar{\rho} \bar{c}}\left(P^{+} e^{-\mathrm{i} k \frac{x}{1+M}}-P^{-} e^{+\mathrm{i} k \frac{x}{1-M}}\right), \\
& \hat{s}(x)=S e^{-\mathrm{i} k_{s} x}
\end{aligned}
$$

where $P^{+}$and $P^{-}$are the complex amplitudes of the downstream and upstream traveling acoustic waves, respectively. The entropy wave with amplitude $S$ is characterized by the entropy wave-number $k_{s}=\omega / \bar{u}$. The propagation matrix $\mathbf{P}$ of a duct module with length $l$ relates the upstream and downstream wave amplitudes, collected in a vector $\mathbf{w}=\left(P^{+}, P^{-}, S\right)^{T}$, to each other: $\mathbf{w}(x+l)=\mathbf{P w}(x)$ with $\mathbf{P}=\operatorname{diag}\left(e^{-i k l /(1+\bar{M})}, e^{+i l x /(1-\bar{M})}, e^{-\mathrm{i} k_{s} l}\right)[1]$.

The flame is modeled as a discontinuity on which the conservation of mass, momentum and energy flow rates are applied. It is a source in the energy flow rate equation which induces frequency-dependent acoustic and entropy fluctuations depending on the volumetric heat release:

$$
\hat{\dot{q}}_{V}(f)=\frac{1}{A \delta_{f}} \dot{Q}(f)
$$

where $A$ and $\delta_{f}$ are the combustor cross-section area and flame thickness, respectively. The flame is placed between $0.01 \mathrm{~m}$ and $0.02 \mathrm{~m}$ in the combustor but its distribution has, however, almost no effect on the resulting pressure spectrum.

For reductions in nozzle cross-sectional area and for gradual area increases, it is assumed that the mean flow does not separate so that isentropic conditions hold. Then, the mean flow quantities upstream and downstream of the area change are related to each other by applying conservation of mass, energy and angular momentum flow rates across the area change.

Concerning the boundary conditions (BCs) the inlet of the center air jet is assumed to be non-reflecting due to the high jet losses implying $Z=1$ in Eq. (7). Different $\mathrm{BCs}(Z \rightarrow \infty$ and $Z=1)$ were tested for the perforated screen because neither experimental reflection coefficients were available nor simple analytical models can be applied. It was found that the perforated screen BC has no noticeable effect on the pressure predicted in the combustor. The swirler is modeled by a short duct with $9 \mathrm{~mm}$ length which is connected to a large plenum, i.e. $\hat{p}=0$ in Eq. (7). The nozzle at the combustor outlet is choked where the Marble \& Candel [36] choked nozzle boundary condition is applied, i.e. $2 \frac{\hat{u}}{\bar{u}}+\frac{\hat{\rho}}{\bar{\rho}}-\frac{\hat{p}}{\bar{p}}=0$.

\subsection{RANS/LNSE-Approach}

In the first step of the hybrid approach RANS simulations are performed to compute both the mean flow field and the combustion noise source term using the statistical combustion noise model. The stationary and compressible RANS equations are closed by the $k-\varepsilon$ turbulence model along with standard wall functions at all walls. The RANS equations 


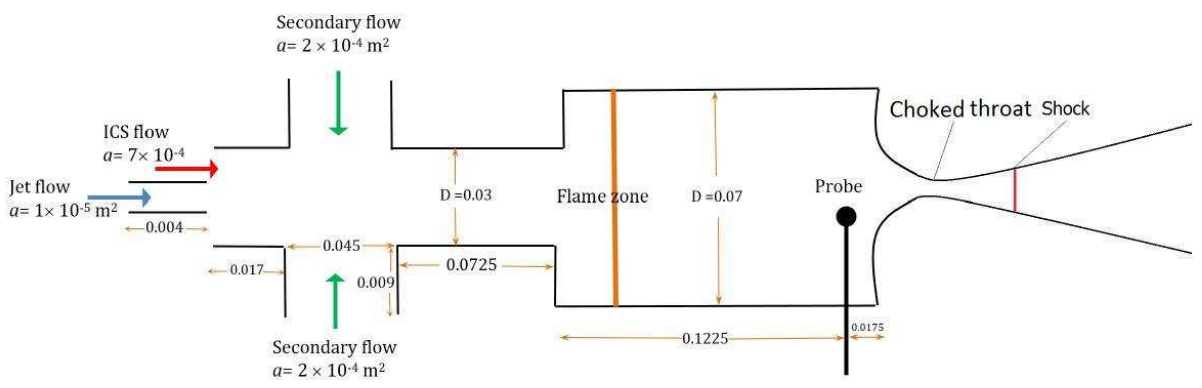

Fig. 2. Acoustic network model for LOTAN solver

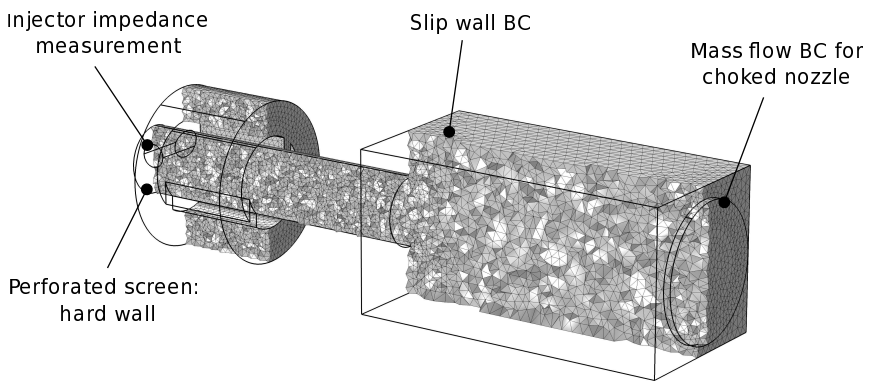

(a) Spatial discretization and boundary conditions

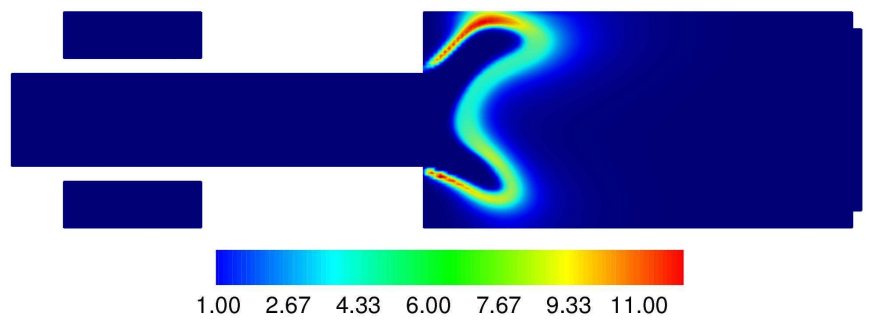

(b) Source distribution $\bar{\rho} \hat{s}_{V}\left[\mathrm{~kg} /\left(\mathrm{sm}^{3}\right)\right]$

Fig. 3. Acoustic LNSE model

are solved numerically using the finite-volume technique on a fully structured grid of 1.12 million cells. Second order upwind discretization schemes are applied for all equations apart from those describing the turbulence where first order upwind schemes are used. The pressure-velocity coupling is calculated by means of the SIMPLE algorithm. The heat of combustion is closed by the finite-rate/Eddy-Dissipation Model (EDM) [37] so that the reaction is approximated by a global reversible one-step chemistry. The high unsteadiness of the flow in the nozzle implies to split up the simulation into a threedimensional reactive combustor simulation and a quasi two-dimensional axisymmetric and non-reactive nozzle simulation. In the 3D combustor simulation the mass flow and total temperature are imposed at the swirler, perforated screen and axial air injection. The nozzle throat at $x=0.173 \mathrm{~m}$ defines a static pressure outlet enforcing 1.07721 bar. Apart from the cooled combustor walls with constant temperature of $\widetilde{T}=303 \mathrm{~K}$, the remaining walls are defined as smooth, no-slip and adiabatic conditions. Once the 3D combustor simulations are finalized the resulting static temperature and velocity distributions are extracted at the nozzle throat plane and prescribed at the inlet at $x=0.173 \mathrm{~m}$ of the quasi $2 \mathrm{D}$ nozzle simulation. The static ambient pressure of 1.01325 bar is defined at the nozzle outlet. Again all walls are represented by smooth, no-slip and adiabatic conditions.

In this study the isentropic formulation of the LNSE simulations is solved since the investigated flame is almost perfectlypremixed. Consequently it neither remarkably features species nor entropy fluctuations. This was recently confirmed by experimental studies [5] so that the isentropic assumption is sufficiently fulfilled. Setting $\hat{s}=0$ in Eq. (5) yields the pressure-density relation $\hat{p}=\bar{c}^{2} \hat{\rho}$ which reduces the number of dependent variables by one and allows to drop the linearized energy Eq. (3). Then combustion noise sources are introduced by the volume source term $\hat{s}_{V}$ in the linearized continuity Eq. (1) [25]. The volume source term and volumetric heat release are related through Eq. (4). With a given integral heat release 


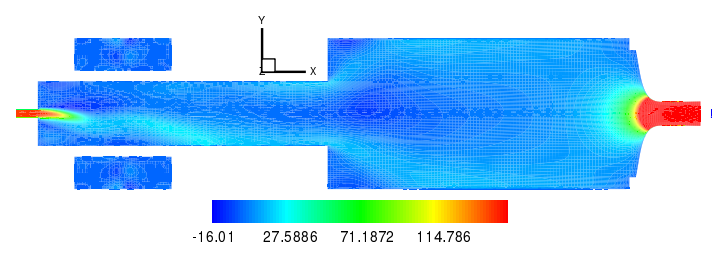

(a) Axial velocity $[\mathrm{m} / \mathrm{s}]$

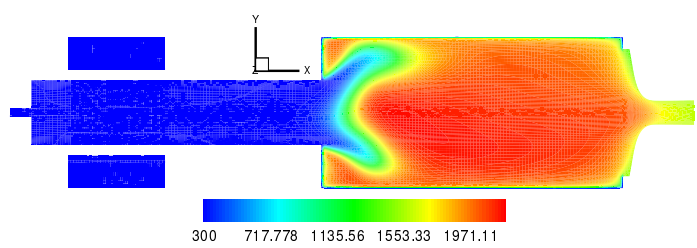

(b) Static temperature $[\mathrm{K}]$

Fig. 4. RANS mean flow fields

spectrum $\dot{Q}(f)$, the combustion noise sources are locally distributed according to the mean heat release $\overline{\dot{\omega}}_{T}$ :

$$
\hat{\dot{q}}_{V}(\boldsymbol{x}, f)=\dot{Q}(f) \frac{\overline{\dot{\omega}}_{T}(\boldsymbol{x})}{\dot{m}_{F} H_{u}},
$$

where $\dot{m}_{F}$ denotes the total fuel mass flow and $H_{u}$ the lower heating value of propane. The resulting distribution of the combustion noise sources - given in terms of $\bar{\rho} \hat{s}_{V}-$ is shown in Fig. 3(b).

The boundary conditions are chosen in accordance with the LOTAN network solver. Therefore the nozzle is again replaced by the chocked mass flow BC along with the isentropic assumption, yielding $2 \frac{\hat{u}}{\bar{u}}-\frac{\hat{\rho}}{\bar{\rho}}(\kappa-1)=0$ and $Z=\frac{2}{(\kappa-1) \bar{M}}$ in Eq. (7), respectively [1,38]. The validity of this model for the nozzle was proven in previous studies [25, 29]. All walls are characterized by slip boundary conditions, i.e. $\hat{\mathbf{u}} \cdot \mathbf{n}=0$, as viscosity can most probably be neglected in the low frequency range. The feeding line and the perforated screen are approximated by a hard wall BC. The LNSE are discretized using the Galerkin/least-squares stabilized finite-element method [39] and solved on an unstructured mesh of 312,613 tetrahedron elements with linear Lagrangian shape functions. The acoustic model and the mesh are shown in Fig. 3(a).

\section{Results}

The presentation of the results is focused on the acoustic part, while the RANS mean flow simulations and a comparison with PIV measurements are only briefly discussed. Further details of the mean flow simulation results can be found in a previous study [25].

\subsection{Mean flow simulations}

In Figs. 4(a) and 4(b) the RANS mean axial velocity and temperature distribution in the $(y, z)$-center-plane are given. Obviously, the jet is deflected off the center axis and the swirling flow in the premixing duct is not axisymmetrical. This leads to a significant deformation of the mean flame shape which burns in the outer shear layer and is strongly deflected towards the lower combustor wall.

In Fig. 5 the RANS simulation results are compared with the particle-image velocimetry (PIV) measurements provided by CNRS [20,21]. The upper plots show the the mean axial velocity profiles over the chamber height for several axial evaluation planes. The corresponding radial velocity profiles are given in the lower plots. The RANS simulations are based on two different meshes with different resolutions and total element numbers of 1.12 and 2.31 million cells. Close to the combustor inlet the qualitative shape of the velocity profiles is captured by the RANS with good agreement. There are moderate deviations with respect to the amplitude of the axial velocity, in particular in the core region of the flow. The deviations between RANS and PIV significantly increase in downstream direction. At the combustor inlet the numerical solution is shown to be only weakly depending on the mesh. The mesh sensitivity drastically increases in downstream direction which is presumably attributed to the highly unsteady flow since the RANS shows an oscillatory behavior with the iteration steps. Nevertheless, the mean flow is predicted with reasonable agreement for this test case.

\subsection{Acoustic simulations}

In a postprocessing step the acoustic pressure is extracted from the numerical solution at the wall at an axial location of $x=122.5 \mathrm{~mm}$ (probe position in Fig. 2) and compared with the experimental measurements conducted by CNRS [20,21]. It should be noted that the measurements were taken at a time where the nozzle was partially eroded which does not have the ideal nozzle shape as assumed by the simulation. All pressure results are given in terms of the Sound Pressure Level (SPL), 

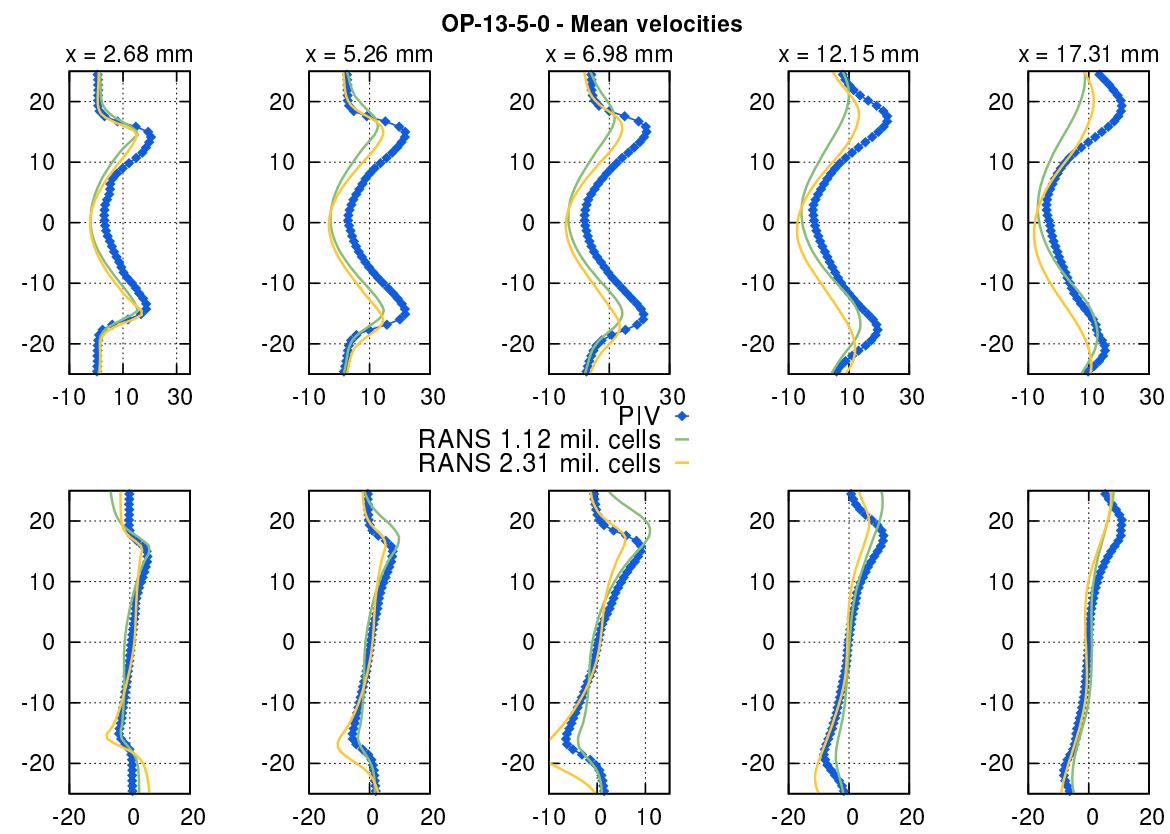

Fig. 5. Comparion PIV measurements $[20,21]$ and RANS results in several axial evaluation planes. Top: Axial velocity [m/s], bottom: radial velocity $[\mathrm{m} / \mathrm{s}]$

computed by

$$
\mathrm{SPL}=20 \log \left(|\hat{p}| / p_{\text {ref }}\right)=20 \log \left(\left|\mathcal{F}\left\{p^{\prime}(t)\right\}\right| / p_{\text {ref }}\right)
$$

In the following the different influences on the noise spectrum are investigated.

\subsubsection{Influence of source model.}

The integral heat release spectra were determined by the spectral noise model, incompressible LES (PRECISE) and compressible LES (AVBP) as shown in Fig. 6. It should be noted that the statistical noise model was only evaluated up to a frequency of $1000 \mathrm{~Hz}$ so that the acoustic simulations are also limited by this frequency for this source model. Obviously, all models predict similar peak frequencies in the range from approximately $120 \mathrm{~Hz}$ up to $150 \mathrm{~Hz}$. However, large differences are observable for the maximum amplitudes at the peak frequency, as well as the slope of the spectrum. While the spectral noise model delivers an absolute amplitude of $550 \mathrm{~W} / \mathrm{Hz}$, the incompressible LES predicts a much higher value of $1550 \mathrm{~W} / \mathrm{Hz}$ and the compressible LES even $7200 \mathrm{~W} / \mathrm{Hz}$. The spectral model gives the lowest spectral decay rates so that the corresponding amplitudes are higher than the LES models above $500 \mathrm{~Hz}$. Both LES models predict similar spectral decay rates. The differences between PRECISE and AVBP codes in predicting the maximum amplitude can be attributed to the influence of the acoustics on the flame as initially discussed in the context of the compressible LES source term extraction. Moreover, different numerical techniques and grid resolutions used in the simulation may lead to the differences in the maximum amplitudes.

The Hirsch/Liu spectral model requires mean flow and turbulent properties such as, velocity, density, turbulent intensity, turbulent dissipation and etc. As Fig. 5 shows RANS cannot well-predict the flow properties and consequently the heat release spectrum using Hirsch/Liu et al. spectral model is not well-predicted in Fig. 6. The capability of the spectral model has been verified in predicting combustion noise for open flames [12] and in gas turbine combustion [1]. Therefore, the authors believe that the discrepancy observed in Fig. 6 might be improved by more accurate RANS, whose accuracy is however limited by the highly unsteady flow.

The different source models directly affect the resulting acoustic pressure spectra in the combustor as demonstrated in Fig. 7 for the LOTAN and LNSE solver. Concerning the LOTAN solution the peak frequency is nicely predicted for all of the three source models. The amplitude and slope are in good agreement with the experimental results when using either the incompressible or compressible LES. There is the tendency of a slight overprediction of the pressure amplitudes when using the compressible LES, but in general the LES models deliver similar results. This leads to the conclusion that the 


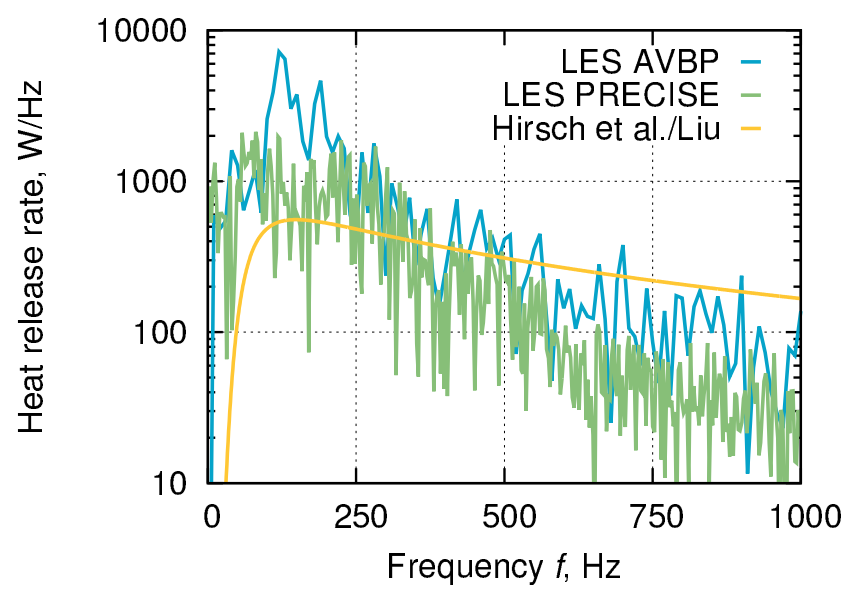

Fig. 6. Integral heat release spectra obtained by different source models
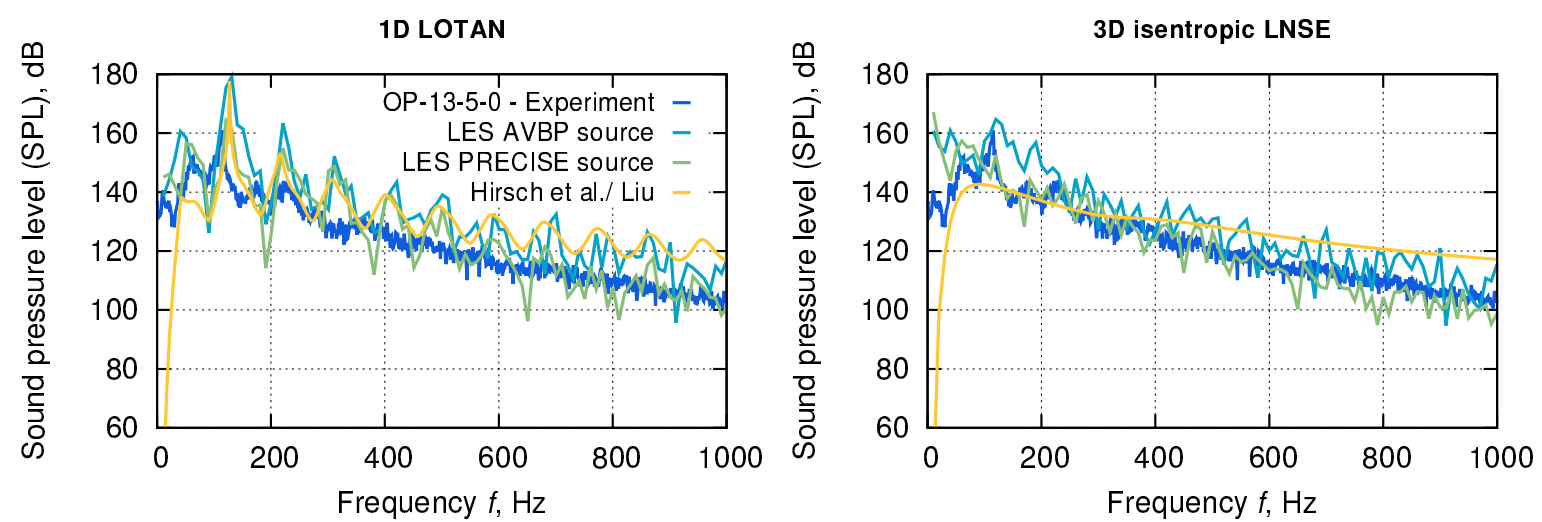

Fig. 7. Pressure spectrum up to $1000 \mathrm{~Hz}$ computed by the LOTAN network solver (left) and RANS/LNSE (right)

incompressible LES is to be preferred, especially when considering the significant save of computational resources and time. As already seen in the source spectrum the application of the statistical noise model results in a well correlated acoustic spectrum with the measurements. However, the trend is not correctly predicted which leads to an overestimation of the pressure amplitudes for higher frequencies above $400 \mathrm{~Hz}$.

Moreover, the effect of the phase of the source term is investigated using the example of the incompressible LES PRECISE source spectrum and the LNSE simulation. The evaluation of the LES delivers a complex spectrum which contains the phase information. The first LNSE simulations were performed using the absolute value of the spectrum $|\dot{Q}(f)|$. A second simulation is performed on basis of the complex spectrum $\dot{Q}(f) \in \mathbb{C}$. The comparison of the simulations is given in Fig. 8. Obviously, there is almost no difference between the absolute and complex spectrum, which demonstrates that the phase relation of the combustion noise is negligible in the low frequency range of interest. Consequently, all further simulations are based on absolute values of the spectrum. Moreover, the effect of the source region on the combustion noise spectrum is investigated in the LNSE simulations by defining a thin flame sheet $\left(\delta_{f}=0.01 \mathrm{~m}\right)$ in the axial range from $0.01 \mathrm{~m}$ to $0.02 \mathrm{~m}$ and exploiting Eq. (14) to compute the volumetric heat release. The corresponding result is referred to as rectangular source region in Fig. 8. Clearly, the shape of the source region does not influence the resulting pressure spectrum in the low frequency range up to $1000 \mathrm{~Hz}$. This is consistent with the observations made with LOTAN and also previous studies [40] and confirms the validity of the compact flame assumption in this range. There is in increasing influence of the source region with increasing frequency, but this effect remains small even at $4000 \mathrm{~Hz}$. Hence, the shape of the source region can be defined arbitrarily as long as the integral fluctuating energy release is conserved. 

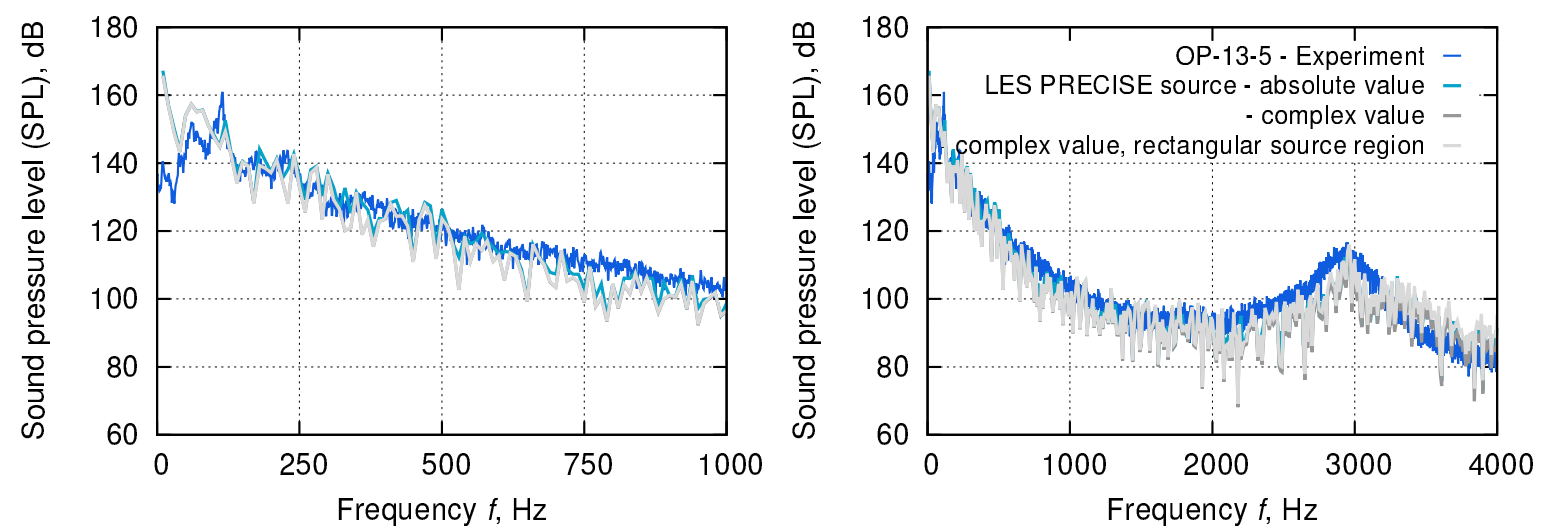

Fig. 8. Influence of the phase and the distribution of the combustion noise source term on the pressure spectrum computed by RANS/LNSE approach (LES PRECISE source term)

\subsubsection{Influence of acoustic propagation solver.}

In the following the LOTAN and LNSE results are compared against each other on the left and right in Fig. 7, respectively. Depending on the source model both solvers deliver similar results in the low frequency range up to $1000 \mathrm{~Hz}$. However, significant differences are observed for very low frequencies below $50 \mathrm{~Hz}$ where the LOTAN solution behaves according to the heat release spectrum and captures the trend in measured pressure fluctuations at low frequencies. This stands in contrast to the LNSE solution which predicts much higher amplitudes at very low frequencies so that the peak amplitude is far less pronounced than in LOTAN. Moreover, the LOTAN solution shows an oscillatory behavior around the trend of the pressure spectrum which is not visible in the LNSE results. This is due to resonances caused by multiple reflections of waves between the choked nozzle and the flame, an effect that is more pronounced for the entropy waves. Resonances in the entropy wave are expected to be related to the sum of the time taken for the waves to travel a distance from combustion zone to nozzle and back. The relevant time is based on convection from the combustion zone to the nozzle $l_{c} / \bar{u}$, and acoustic propagation back upstream $l_{c} /(\bar{c}-\bar{u})$. For a low Mach number mean flow this cycle time is approximately $l_{c} / \bar{u}$ leading to a very much smaller frequency interval $\bar{u} / l_{c}$, that is about $100 \mathrm{~Hz}$ in Fig. 7 . It should be pointed out that the dissipation of entropy waves is not included in the LOTAN modeling, which is likely to reduce the effect of resonance. Hence the amplitude of the peaks decreases particularly at higher frequencies if the dissipation of entropy noise is included in LOTAN.

An acoustic combustor eigenfrequency at about $3000 \mathrm{~Hz}$ is not captured by LOTAN, cf. Fig. 9. This stands in contrast to the LNSE solution where the first eigenfrequency is predicted. However, a second eigenfrequency is visible at approximately $3300 \mathrm{~Hz}$ which is not measured in the experiment. The LNSE predicts smaller pressure amplitudes as compared with LOTAN which increases with frequency, which might be due to a higher damping included in the model.

It is concluded that the influence of the acoustic solver in the low frequency regime up to $1000 \mathrm{~Hz}$, where the combustion noise is important, is fairly weak. In this range the shape of the spectrum is mainly controlled by the heat release source term. Both the LOTAN and LNSE solver are able to predict the pressure spectrum with good agreement in terms of amplitude and trend. This also demonstrated that a quasi one-dimensional model is sufficient to describe the acoustics in the low frequency range. However, the LOTAN solver shows an oscillatory behavior due to resonances triggered by the entropy wave. In practice, these are attenuated at high frequency due to the effect of entropy dissipation that is not included in this version of the LOTAN code. This represents an on-going research by the authors.

Finally, the analysis allows to draw some conclusions concerning the source term implementations. The LOTAN simulation is based on the heat of combustion source term $\hat{\dot{q}}_{V}$ in the energy Eq., whereas the LNSE simulations rely on the volume source term $\hat{s}_{V}$ in the continuity Eq. Consequently the analysis confirmed the validity of the volume source term model, Eq. (4), applied in the LNSE simulations.

\subsubsection{Influence of mean flow.}

Finally, the influence of the aerodynamic mean flow convection and shearing on the direct combustion noise spectrum is separated by performing a second simulation based on the inhomogeneous Helmholtz Eq. (6). The same impedance boundary conditions as in the LNSE simulations were imposed, but rewritten in terms of the acoustic pressure utilizing Eq. (8). 

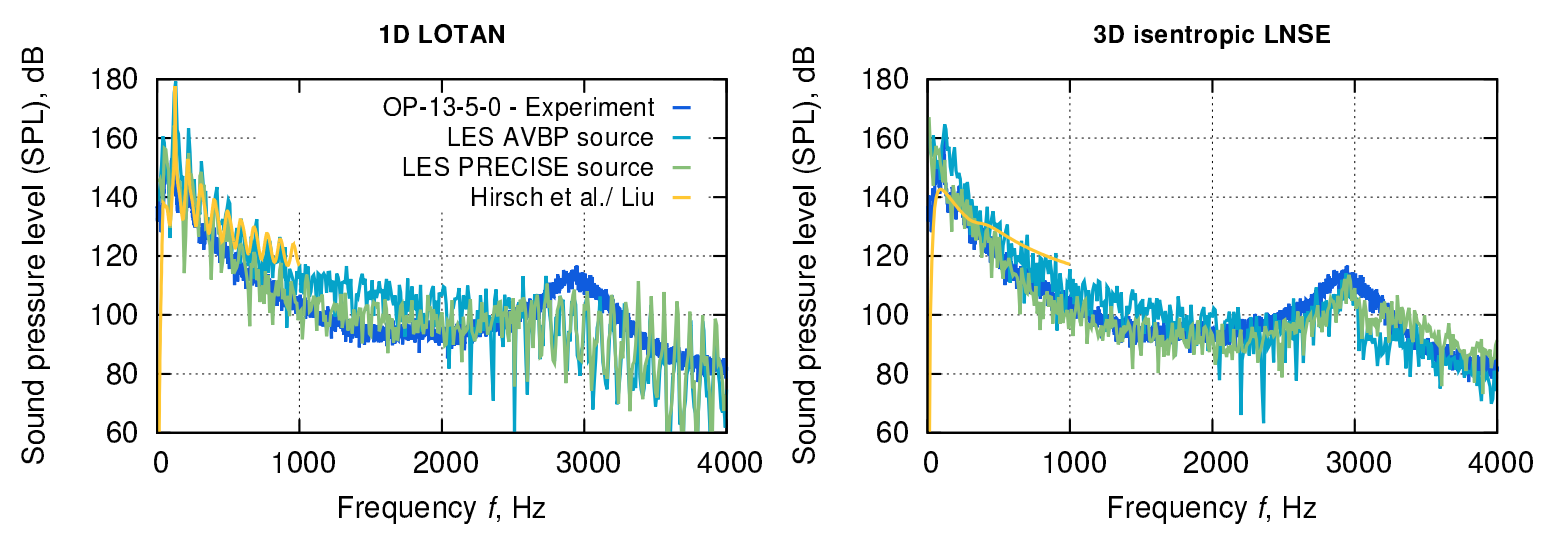

Fig. 9. Pressure spectrum up to $4000 \mathrm{~Hz}$ computed by the LOTAN network solver (left) and RANS/LNSE (right)
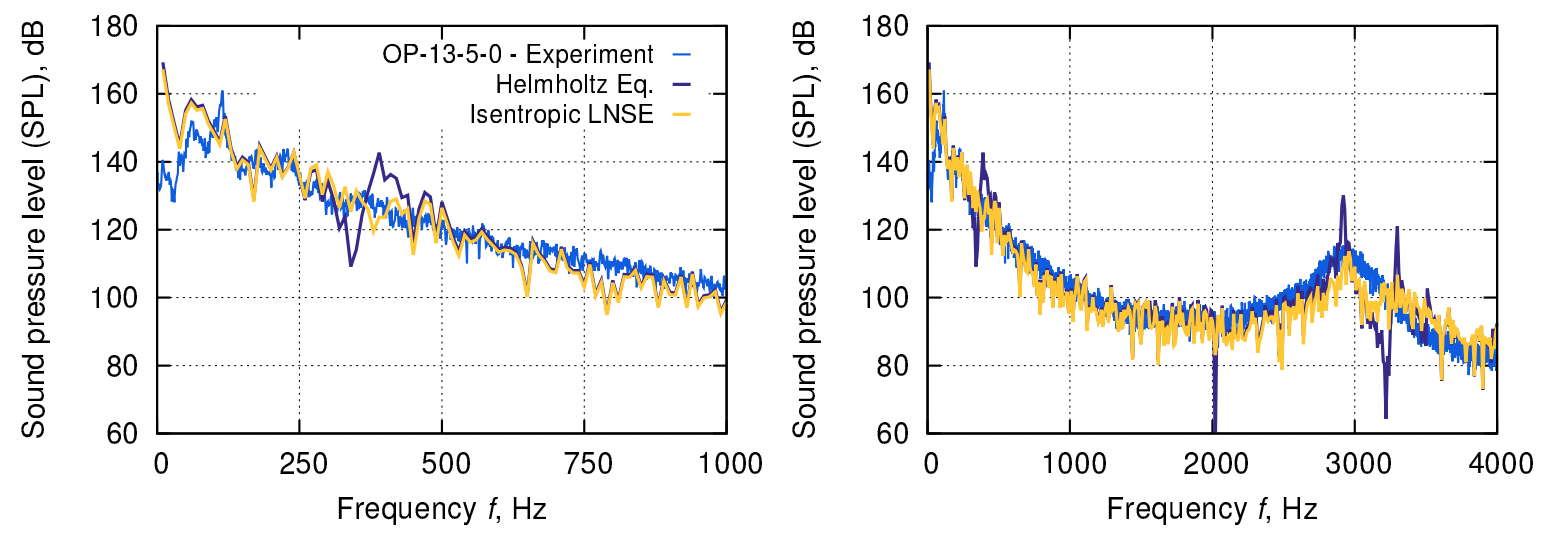

Fig. 10. Influence of the aerodynamic mean flow on the combustion noise spectrum (LES PRECISE source term)

The pressure spectrum obtained by the Helmholtz Eq. is shown in Fig. 10 and compared with the corresponding LNSE simulations. The simulations are based on the incompressible LES PRECISE source term while keeping in mind that the other source models deliver very similar results. Obviously, it can be seen that the Helmholtz Eq. predicts almost the same broadband pressure spectrum. The LNSE solution features slightly lower pressure amplitudes which is presumably due to the viscous and shear flow damping. It can be concluded that in the present test case the influence of the aerodynamic flow field on the broadband combustion noise contribution is negligible. This result also further confirms the validity of the simplifications made with LOTAN where three-dimensional mean flow effects are not included.

However, the combustor eigenfrequencies are significantly affected by the aerodynamic mean flow which is explained in the following. The Helmholtz Eq. predicts the same acoustic eigenfrequencies as compared with the LNSE simulation at approximately $3000 \mathrm{~Hz}$ and $3300 \mathrm{~Hz}$, but the amplitudes are significantly overpredicted. Furthermore, another eigenfrequency at $390 \mathrm{~Hz}$ is visible when using the Helmholtz Eq. which is not observed in the experiment. This eigenfrequency corresponds to the $\lambda / 2$-mode in premixing duct and combustion chamber together. Much better amplitude predictions at the different eigenfrequencies are achieved when using the LNSE due to the incorporation of damping effects related to the interaction of the acoustic waves with the mean flow. The first eigenfrequency at $390 \mathrm{~Hz}$ is completely damped if the LNSE model is applied on the combustor. In conclusion, the analysis showed that the LNSE or LEEs should be used to obtain realistic complex eigenfrequencies $\omega=\omega_{r}+\mathrm{i} \omega_{i}$, in which the damping rate is characterized by the imaginary part $\omega_{i}[30]$. Hence, we expect the Helmholtz and LNSE solver to deliver similar real parts of the eigenfrequencies $\omega_{r}$, but very different imaginary parts $\omega_{i}$ and damping rates, respectively. An eigenfrequency analysis of the combustor should be addressed in further studies.

\section{Conclusions}

In the current study the low-order network solver LOTAN and the hybrid RANS/LNSE-approach are applied on the generic CESAM-HP combustor. Each solver is fed by three different combustion noise terms obtained from a statistical 
noise model, the incompressible LES solver PRECISE-UNS and compressible LES solver AVBP, respectively. In this way the influence of the source model and solver are identified for one out of three operating points which supports the most stable combustion conditions and is less affected by thermoacoustic instabilities. The analysis showed that:

1. the LOTAN and LNSE solver deliver similar results for the different source term models which demonstrates that the noise spectra are mainly determined by the heat release source spectrum in the low frequency range,

2. the compressible LES source term does not lead to better results as compared with the incompressible LES which is therefore to be preferred for the source term computation,

3. the LOTAN network solver is more appropriate for the early combustor design stage, whereas LNSE simulations and incompressible LES sources should be favored at an advanced stage,

4. the phase distribution and shape of the source region is of minor importance for the resulting noise spectrum, and

5. the influence of the aerodynamic mean flow on the broadband combustion noise spectrum is negligible.

Future studies will be necessary to evaluate the effect of the entropy wave generation and propagation on the indirect noise contribution to the pressure spectrum. This can only be achieved by applying either LOTAN or LNSE which includes the attenuation of entropy wave propagation.

\section{Acknowledgements}

The authors gratefully acknowledge the financial support provided by the European Union in the Seventh Framework Programme FP7 under Grant Agreement No. ACP2-GA-2012-312444, the project RECORD (Research on Core Noise Reduction) and by the German Research Foundation (DFG) in the combustion noise project (JA 544/37-2). The authors also address special thanks to Sébastien Ducruix and Marek Mazur from CNRS for providing the pressure and PIV data.

\section{References}

[1] Dowling, A., and Mahmoudi, Y., 2015. “Combustion noise”. Proceedings of the Combustion Institute, 35, pp. 65-100.

[2] Liu, T., Dowling, A., Swaminathan, N., Morvant, R., Macquisten, M., and Caracciolo, L., 2013. "Prediction of combustion noise for an aeroengine combustor". Journal of Propulsion and Power, 30(1), pp. 114-122.

[3] Howe, M., 2010. “Indirect combustion noise”. Journal of Fluid Mechanics, 659, pp. 267-288.

[4] Cumpsty, N., 1979. "Jet engine combustion noise: Pressure, entropy and vorticity perturbations produced by unsteady combustion or heat addition”. Journal of Sound and Vibration, 66(4), pp. 527-544.

[5] Kings, N., Tao, W., Scouflaire, P., Richecoeur, F., and Ducruix, S., 2016. "Experimental and numerical investigation of direct and indirect combustion noise contributions in a lean premixed laboratory swirled combustor". In Proceedings of ASME Turbo Expo 2016, no. GT2016-57848.

[6] Livebardon, T., Moreau, S., Poinsot, T., and Bouty, E., 2015. "Numerical investigation of combustion noise generation in a full annular combustion chamber". In 21th AIAA/CEAS Aeroacoustics Conference.

[7] Ewert, R., and Schröder, W., 2003. "Acoustic perturbation equations based on flow decomposition via source filtering". Journal of Computational Physics, 188, Mar., pp. 365-398.

[8] Bui, T., Schröder, W., and Meinke, M., 2007. "Acoustic perturbation equations for reacting flows to compute combustion noise". International Journal of Aeroacoustics, 6(4), pp. 335-355.

[9] Mühlbauer, B., Ewert, R., Kornow, O., and Noll, B., 2010. "Evaluation of the rpm approach for the simulation of broadband combustion noise". AIAA Journal, 48(7), July.

[10] Grimm, F., Noll, B., Aigner, M., Ewert, R., and Dierke, J., 2014. "The fast random particle method for combustion noise prediction". In 20th AIAA/CEAS Aeroacoustics Conference.

[11] Ewert, R., 2006. "Broadband slat noise prediction based on caa and stochastic sound sources from a fast random particle-mesh (rpm) method". In 12th AIAA/CEAS Aeroacoustics Conference.

[12] Hirsch, C., Waesle, J., Winkler, A., and Sattelmayer, T., 2006. "A spectral model for the sound pressure from turbulent premixed combustion". In 31st International Symposium on Combustion, pp. 1435-1445.

[13] Hirsch, C., Wäsle, J., Winkler, A., and Sattelmayer, T., 2007. "A spectral model for the sound pressure from turbulent premixed combustion". Proceedings of the Combustion Institute, 31, pp. 1435-1441.

[14] Jörg, C., 2015. "Experimental Investigation and Spectral Modeling of Turbulent Combustion Noise from Premixed and Non-premixed Flames". PhD thesis, Technische Univeristät München.

[15] Anand, M. S., Eggels, R., Staufer, M., Zedda, M., and Zhu, J., 2013. "An advanced unstructured-grid finite-volume design system for gas turbine combustion analysis". ASME 2013 Gas Turbine India Conference, GTINDIA 2013, D, pp. 1-12.

[16] Raynaud, F., 2015. “Towards unsteady simulation of combustor-turbine interaction using an integrated approach". In Proceedings of ASME Turbo Expo 2015, pp. 1-11.

[17] Cerfacs, 2008. The AVBP HandBook (2008). 
[18] Lapeyre, C. J., Mazur, M., Scouflaire, P., Richecoeur, F., Ducruix, S., and Poinsot, T., 2016. "Acoustically Induced Flashback in a Staged Swirl-Stabilized Combustor". Flow, Turbulence and Combustion, June.

[19] Huet, M., Vuillot, F., Bertier, N., Mazur, M., Kings, N., Tao, W., Scouflaire, P., Richecoeur, F., Ducruix, S., Lapeyre, L., and Poinsot, T., 2016. "Recent improvements in combustion noise investigation: from combustion chamber to nozzle flow". AerospaceLab Journal, June.

[20] Mazur, M., Scouflaire, P., Richecoeur, F., and Ducruix, S., 2015. "Combustion noise studies of a swirled combustion chamber with a choked nozzle using high-speed diagnostics". In ANERS 2015 / XNOISE conference.

[21] Kings, N., Mazur, M., Tao, W., Scouflaire, P., Richecoeur, F., and Ducruix, S., 2015. "Experimental and numerical investigation on combustion noise sources in a choked model gas turbine combustor". In ANERS 2015 / XNOISE conference.

[22] Gikadi, J., Sattelmayer, T., and Peschiulli, A., 2012. "Effects of the mean flow field on the thermo-acoustic stability of aero-engine combustion chambers".

[23] Gikadi, J., 2013. "Prediction of Acoustic Modes in Combustors using Linearized Navier-Stokes Equations in Frequency Space". PhD thesis, Technische Univeristät München, Dezember.

[24] Ullrich, W., and Sattelmayer, T., 2015. "Transfer functions of acoustic, entropy and vorticity waves in an annular model combustor and nozzle for the prediction of the ratio between indirect and direct combustion noise". In 21 th AIAA/CEAS Aeroacoustics Conference.

[25] Ullrich, W., Hirsch, C., and Sattelmayer, T., 2016. "Computation of combustion noise from a premixed and pressurized propane flame using statistical noise modeling". In Proceedings of the AIAA Propulsion and Energy Forum and Exposition (Propulsion and Energy 2016), no. AIAA-2016-4590.

[26] Strahle, W., 1972. "Some results in combustion generated noise". Journal of Sound and Vibration, 23(1), pp. 113-125.

[27] Chu, B., and Kovsznay, L., 1957. "Non-linear interactions in a viscous heat-conducting compressible gas". Combustion and Flame.

[28] Poinsot, T., and Veynante, D., 2005. Theoretical and Numerical Combustion, 0002 ed. RT Edwards Inc, Jan.

[29] Ullrich, W., Lackhove, K., Fischer, A., Hirsch, C., Sattelmayer, T., Sadiki, A., and Staufer, M., 2016. Combustion noise prediction using linearized navier-stokes equations and incompressible les sources. Submitted to Journal of Propulsion and Power.

[30] Dowling, A., and Stow, S., 2003. "Acoustic analysis of gas turbine combustors". AIAA Journal of Propulsion and Power, 19(5), Oct.

[31] Mazur, M., Tao, W., Scouflaire, P., Richecoeur, F., and Ducruix, S., 2015. "Experimental and analytical study of the acoustic properties of a gas turbine model combustor with a chocked nozzle". In Proceedings of ASME Turbo Expo 2015, no. GT2015-43013.

[32] Stow, S., and Dowling, A., 2001. "Thermoacoustic oscillations in an annular combustor". In Proceedings of ASME Turbo Expo 2001, no. 2001-GT-0037.

[33] Stow, S., and Dowling, A., 2009. "A time-domain network model for nonlinear thermoacoustic oscillations". ASME Journal of Engineering for Gas Turbines and Power, 131(3).

[34] Mahmoudi, Y., Dowling, A., and Stow, S., 2015. "Direct and indirect combustion noise in an idealised combustor". In 25th International Colloquium on Dynamic of Explosions and Reactive Systems (ICDERS).

[35] Nicoud, F., and Wieczorek, K., 2008. "About the zero mach number assumption in the calculation of thermoacoustic instabilities". AIAA Journal.

[36] Marble, F., and Candel, S., 1977. "Acoustic disturbances from gas non-uniformities convected through a nozzle". Journal of Sound and Vibration, 55(2), pp. 225-243.

[37] ANSYS, 2011. ANSYS FLUENT Theory Guide, Nov.

[38] Stow, S., Dowling, A., and Hynes, T., 2002. "Reflection of circumferential modes in a choked nozzle". Journal of Fluid Mechanics, 467, Sept., pp. 215-239.

[39] Hughes, T., Franca, L., and Hulbert, G., 1989. "A new finite element formulation for computational fluid dynamics: Viii. the galerkin/least-squares method for advective-diffusive equations". Computational Methods in Applied Mechanical Engineering, 73, pp. 173-189.

[40] Weyermann, F., 2010. "Numerische berechnung der emission verbrennungsinduzierten lärms automobiler zusatzheizungen". PhD thesis, Technische Universität München, Sept. 